\title{
Effects of diazepam on BOLD activation during the processing of aversive faces
}

\author{
Cristina M Del-Ben ${ }^{1}$, Cesar AQ Ferreira ${ }^{1}$, Tiago A Sanchez ${ }^{2}$, \\ Wolme C Alves-Neto ${ }^{1}$, Vinicius G Guapo ${ }^{1}$, Draulio B de Araujo ${ }^{2,3,4}$ \\ and Frederico G Graeff ${ }^{1}$
}

Psychopharm

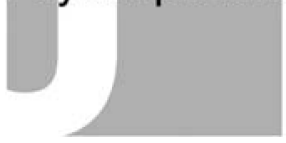

Journal of Psychopharmacology 26(4) 443-451

(c) The Author(s) 2012

Reprints and permissions: sagepub.co.uk/journalsPermissions.nav DOI: $10.1177 / 0269881110389092$ jop.sagepub.com

@SAGE

\begin{abstract}
This study aimed to measure, using fMRI, the effect of diazepam on the haemodynamic response to emotional faces. Twelve healthy male volunteers (mean age $=24.83 \pm 3.16$ years), were evaluated in a randomized, balanced-order, double-blind, placebo-controlled crossover design. Diazepam $(10 \mathrm{mg})$ or placebo was given $1 \mathrm{~h}$ before the neuroimaging acquisition. In a blocked design covert face emotional task, subjects were presented with neutral (A) and aversive (B) (angry or fearful) faces. Participants were also submitted to an explicit emotional face recognition task, and subjective anxiety was evaluated throughout the procedures. Diazepam attenuated the activation of right amygdala and right orbitofrontal cortex and enhanced the activation of right anterior cingulate cortex (ACC) to fearful faces. In contrast, diazepam enhanced the activation of posterior left insula and attenuated the activation of bilateral ACC to angry faces. In the behavioural task, diazepam impaired the recognition of fear in female faces. Under the action of diazepam, volunteers were less anxious at the end of the experimental session. These results suggest that benzodiazepines can differentially modulate brain activation to aversive stimuli, depending on the stimulus features and indicate a role of amygdala and insula in the anxiolytic action of benzodiazepines.
\end{abstract}

\section{Keywords}

Benzodiazepines, emotions, facial expression, magnetic resonance imaging

\section{Introduction}

The brain structures underlying defensive behaviour have been implicated in the pathophysiology of anxiety disorders (McNaughton and Corr, 2004). Briefly, it has been proposed that the amygdala and the prefrontal cortex play a key role in risk-assessment behaviour elicited by a potential threat, whereas caudal brain structures, such as the midbrain periaqueductal gray matter, are involved in fight-or-flight reactions to a proximal threat. The former behaviour has been related to generalized anxiety disorder (GAD), and the latter has been related to panic disorder (PD) (Deakin and Graeff, 1991; Graeff and Del-Ben, 2008). In particular, the amygdala has been largely implicated in the processing of threatening stimuli (Davis and Whalen, 2001), among which are emotional faces.

The identification of basic emotions in facial expressions is crucial for social adaptation. However, the adaptive meaning of the perception of each facial expression is not completely understood. It has been suggested that fearful faces could be considered an ambiguous stimulus (Whalen, 1998), indicating a potential threat in the environment. On the other hand, an angry face directed toward a particular individual could represent a proximal threat. If this suggestion, and the predictions derived from animal studies are correct, we would expect that fearful, but not angry faces would provoke neuronal responses in the amygdala. However, whereas neuroimaging studies of healthy volunteers have provided evidence of amygdala activation to both fearful (Breiter et al., 1996; Fitzgerald et al., 2006) and angry (Fitzgerald et al., 2006; Hariri et al., 2000; Nomura et al., 2004) faces, a comparative study has shown that neuronal responses of the amygdala were more pronounced to fearful than to angry faces (Costafreda et al., 2008; Whalen et al., 2001).

Similarly, recently reviewed evidence of the serotonergic modulation of the accuracy in the identification of facial expressions of fear by healthy volunteers suggests that fearful faces are processed as a potential threat. However, no conclusion could be drawn regarding angry faces, because most

${ }^{1}$ Division of Psychiatry, Faculty of Medicine of Ribeirão Preto, University of São Paulo, Brazil

${ }^{2}$ Department of Physics and Mathematics, Faculty of Philosophy, Sciences and Arts of Ribeirão Preto, University of São Paulo, Brazil

${ }^{3}$ Onofre Lopes University Hospital, Federal University of Rio Grande do Norte (UFRN), Brazil

${ }^{4}$ Edmond and Lily Safra International Institute of Neuroscience of Natal (ELS-IINN), Brazil

\section{Corresponding author:}

Cristina M Del-Ben, Departamento de Neurociências e Ciências do Comportamento, Faculdade de Medicina de Ribeirão Preto-USP, Avenida Bandeirantes 3900, CEP: 14048-900, Ribeirão Preto, SP, Brazil

Email: delben@fmrp.usp.br 
of the reviewed studies found no effect of serotonergic drugs on the processing of this facial expression (Del-Ben et al., 2008).

Although benzodiazepines are typical anxiolytic drugs, few studies have investigated their effect on the processing of facial expressions. These have shown seemingly contradictory results. Blair and Curran (1999) showed that $15 \mathrm{mg}$ of diazepam selectively impaired the identification of angry faces. In a further study, however, the same research group reported that the same dose of diazepam affected the perception of both angry and fearful faces (Zangara et al., 2002). To explain these conflicting results, the authors suggested that the emotional state of the volunteers could have interfered with the processing of emotional cues, as participants reported more anxiety and discomfort in the former study than in the latter. Another study pointed to global impairment by diazepam of the identification of emotional faces (Coupland et al., 2003). Other evidence shows that lorazepam (Kamboj and Curran, 2006) or a low dose of diazepam (Murphy et al., 2008) has no effect on the recognition of facial emotional expressions.

The anxiolytic action of the benzodiazepines is due to facilitation of the neurotransmission mediated by gammaamino butyric acid (GABA), which is the main inhibitory neurotransmitter of the central nervous system (CNS) of vertebrates. The main types of GABA receptors are the $\mathrm{GABA}_{\mathrm{A}}$ and $\mathrm{GABA}_{\mathrm{B}}$, which are selectively distributed in the CNS. The $\mathrm{GABA}_{\mathrm{A}}$ and benzodiazepine receptors are associated with a chloride channel, forming a macromolecular complex. Through cooperative allosteric interaction, benzodiazepine agonists increase the affinity of GABA for the $\mathrm{GABA}_{\mathrm{A}}$ receptor. The $\alpha_{2}$ and $\alpha_{3}$ subunits of the GABA receptor, which are responsible for the anxiolytic effects of the benzodiazepines, are located in amygdalar and cortical regions (D'Hulst et al., 2009). In the neocortex, $\mathrm{GABA}_{\mathrm{A}}$ receptors reach high densities in primary sensory areas (Zilles and Amunts, 2009). In the amygdala, benzodiazepine receptor density is higher in the phylogenetically more recent basolateral region than in the more primitive corticomedial area (Niehoff and Kuhar, 1983). High levels of benzodiazepines receptors are also found in hippocampus, subiculum, and ventromedial hypothalamus (Young et al., 1981).

The association of pharmacological challenges with functional magnetic resonance imaging (fMRI) allows the study of drug effects on cerebral metabolism during the performance of specific tasks. To our knowledge, only one study has previously aimed to verify the effects of a benzodiazepine on brain activation in response to emotional faces (Paulus et al., 2005). The results showed that lorazepam attenuates, in a dose-dependent manner, the activation of the amygdala and the insula to emotional faces (happy, angry and fearful), in contrast with a sensorimotor task.

In general, the first choice for the treatment of both GAD and $\mathrm{PD}$ is a selective serotonin reuptake inhibitor (SSRI); however, benzodiazepines are also effective for the treatment of both GAD and PD, although patients with PD seem to have a relative lack of sensitivity to benzodiazepines, with clinical response limited to high-potency benzodiazepines, such as alprazolam and clonazepam (Nutt, 2005). If the hypothesis of fearful and angry faces representing, respectively, potential (related to GAD) and proximal (related to PD) threat is correct, the amygdala should be activated by fearful, but not angry faces, and diazepam should attenuate the activation of the amygdala to fearful, but not angry faces.

To test these predictions, the present study used fMRI to measure the effect of diazepam at a lower than sedative dose on the activation of the amygdala to fearful and angry faces. To be thorough, other brain areas that are related to threat processing and have direct or indirect neural connections with the amygdala were also included in the neuroimaging analyses. Finally, a behavioural task was performed immediately after the scans in order to verify whether diazepam would selectively impair the identification of fearful faces.

\section{Methods \\ Participants}

Twelve healthy male volunteers aged between 19 and 31 years (mean $=24.83, \mathrm{SD}=3.16$ years) took part in this study. Participants were evaluated by the Structured Clinical Interview for DSM-IV, clinical version (SCID-CV) (First et al., 1997), translated into Portuguese (Del-Ben et al., 2001) to exclude current and past psychiatric diagnoses. General medical conditions were excluded by clinical history, physical examination and blood tests. The volunteers were free of any medication for at least 2 months before the procedures. Mean trait anxiety, measured by the State-Trait Anxiety Inventory-Trait form (STAI-T) (Spielberger, 1983) was 34.50 ( $\mathrm{SD}=8.37$ ), varying from 23 to 48 . Female volunteers were not included to avoid possible influences of the phases of the menstrual cycle in the results. The local ethical committee approved this study and written informed consent was obtained from all volunteers.

\section{Psychological measures}

Anxiety and other subjective states were evaluated by the Visual Analogue Mood Scale - VAMS (Norris, 1971) translated into Portuguese (Zuardi and Karniol, 1981). This scale is composed of 16 items, and for each item the participant was asked to mark a point on a $100 \mathrm{~mm}$ line between two adjectives with opposite meanings to describe his current feelings (Zuardi et al., 1993). The test items are grouped into four factors: anxiety, sedation, cognitive impairment and discomfort (Parente et al., 2005). Physical manifestations of anxiety were recorded by the Bodily Symptoms Scale - BSS (Zuardi et al., 1993). The intensity of each symptom was graded from 0 (no symptom) to 4 (highest). The BSS total score was the sum of the values of all of the items for each participant.

\section{Experimental paradigms}

fMRI task. In a blocked design task, subjects were presented with pictures of neutral (A) and aversive (B) (angry or fearful - in two different runs) faces from the Pictures of Facial Affect Series (Ekman and Friesen, 1976). All the volunteers were submitted to both runs in a balanced order, with a rest 
interval of about $5 \mathrm{~min}$ between runs. The blocks lasted $30 \mathrm{~s}$ in an $\mathrm{ABABABABA}$ design for each aversive emotion. Wholebrain images were acquired in a randomized order over $4.5 \mathrm{~min}$ for each emotion. Subjects were asked to identify the gender of the faces, not to describe or focus on the emotion pictured.

Behavioural task. The task consisted of six basic emotions (anger, disgust, fear, sadness, surprise and happiness) from the Pictures of Facial Affect Series (Ekman and Friesen, 1976). For each emotion, 40 pictures, 20 male and 20 female faces, were presented. Faces had been morphed between neutral $(0 \%)$ and each standard emotion $(100 \%)$ in $10 \%$ steps of emotion intensity, comprising four stimuli at each intensity level for each emotion. The faces were presented on a computer screen for $0.5 \mathrm{~s}$, with an interval (blank screen) of $4.5 \mathrm{~s}$ between consecutive stimuli. Volunteers were asked to choose the response that best described the emotion in the picture and to register their responses as soon as possible by pressing one of the labelled keys on the keyboard.

\section{Procedures}

Participants were evaluated on two occasions separated by at least 1 week $($ mean $=4.5, \mathrm{SD}=3.58)$ using a randomized, balanced-order, double-blind, placebo-controlled crossover design. They arrived at the laboratory between 9 am and $12 \mathrm{pm}$ after a light breakfast. Initial subjective measures (VAMS and BSS) were taken $15 \mathrm{~min}$ after arriving and before the capsule intake. Pre-scanner subjective measures were assessed $60 \mathrm{~min}$ later, followed by the neuroimaging acquisition, which lasted around $45 \mathrm{~min}$. Immediately after the image acquisition and just before starting the behavioural task, the post-scanner subjective measures were taken. The behavioural task lasted $30 \mathrm{~min}$, and at the end of the experimental session a final subjective set of measures was registered.

An oral dose of diazepam $(10 \mathrm{mg})$ or placebo was given $1 \mathrm{~h}$ before the beginning of the neuroimaging acquisition. This time interval was chosen considering that although diazepam has a long half-life $(20-70 \mathrm{~h})$, its effective plasma concentration falls between 1 and $2 \mathrm{~h}$ after the drug intake, since diazepam is highly lipid soluble, undergoes redistribution among bodily compartments, and readily binds to plasma protein (Greenblatt and Shader, 1987). Five volunteers were given placebo in the first experimental session and diazepam in the second experimental session, and seven volunteers were given diazepam in the first experimental session.

\section{Image acquisition}

Images were acquired in a $1.5 \mathrm{~T}$ scanner (Siemens, Magneton Vision) with a TX-RX head coil. Echo-planar imaging (EPI) was used to produce 54 contiguous volumes, and each consisted of 16 axial slices (slice thickness $=6 \mathrm{~mm}$; TR $=5000 \mathrm{~ms}$; $\mathrm{TE}=40 \mathrm{~ms}$; flip angle $90^{\circ} ; 64 \times 64$ matrix; FOV $=220 \mathrm{~mm}$, dimension of the voxel $=3.44 \times 3.44 \times 6.00 \mathrm{~mm}$ ). A T1-weighted structural scan (slice thickness $=1 \mathrm{~mm}$;
$\mathrm{TR}=9.7 \mathrm{~ms} ; \mathrm{TE}=4 \mathrm{~ms} ;$ flip angle $12^{\circ} ;$ matrix $256 \times 256$; $\mathrm{FOV}=256 \mathrm{~mm}$ ) was also acquired for each subject for co-registration and in order to exclude any structural abnormality. No abnormality was reported for any of the 12 subjects.

\section{Data analysis}

Imaging data were analysed in Brain Voyager 4.96 (Brain Innovation, Maastricht, The Netherlands), using a general linear model (GLM) with fixed effects model. The dataset was corrected for motion and slice timing, and it was spatially filtered (10 mm FWHM) and temporally filtered (high pass filter of $0.01 \mathrm{~Hz}$ ). Individual functional maps were normalized into the Talairach space (Talairach and Tournoux, 1988). After pre-processing, first-level analysis was performed on each subject using the GLM with a delayed boxcar waveform to model Blood Oxygenation Level Dependent (BOLD) signal changes. After transformation into Talairach space, the group analysis model included four orthogonal contrasts: fearful or angry faces with placebo intake versus neutral faces with placebo; fearful or angry faces with diazepam intake versus neutral faces with diazepam. The main effect of the task was evaluated using a whole-brain approach, which included only images obtained within the placebo session (supplemental material). The effect of the treatment for each emotion was evaluated by contrasting the diazepam versus the placebo conditions, using a whole-brain approach for each emotion, separately (supplemental material). Negative statistical contrast values correspond to attenuation of the response, and positive values were considered as increase in the response amplitude, both promoted by the drug. The a priori hypothesis involved the effects of diazepam on the activation of the amygdala to aversive faces; therefore, this structure was chosen as a region of interest.

We also included in the analyses the following brain structures implicated in the emotional processing of aversive stimuli: the lateral orbitofrontal cortex (Brodmann Area, (BA) 47), the anterior cingulate gyrus (BA 32) and the insula (BA 13). All these structures were selected using their anatomical location. The statistical threshold was set to $p<0.05$ corrected for multiple comparisons (FDR-corrected), and only clusters of at least $50 \mathrm{~mm}^{3}$ were segregated. We have also performed an analysis of covariance (ANCOVA) in BrainVoyager to calculate correlations among activations in the regions of interest where an effect of the drug had been observed in both contrasts: fearful $>$ neutral faces and angry $>$ neutral faces. We have also looked for correlation between the response amplitude obtained under these two conditions and: (1) personality traits of anxiety measured through the STAI-T; (2) the level of anxiety just before the scanner measured by the VAMS anxiety factor; and (3) the accuracy in the behavioural task to recognize fearful and angry faces, respectively. The correlation coefficient $r^{2}$ and its corresponding $p$-value were calculated for each region of interest.

Behavioural and subjective data were analysed using the SPSS statistical package version 13.0. The accuracy of the performance on the behavioural task was defined by the sum of correct answers at all levels of intensity for each 
emotion in male and female faces, divided by the total number of possible correct answers for the same emotion in each sex (20 female faces and 20 male faces for each emotion). Accuracy was analysed using MANOVA with repeated measures (Hotelling's Trace) and with the following as factors: emotion (anger, disgust, fear, sadness, surprise and happiness), sex of the faces (female and male) and treatment (diazepam or placebo). Post-hoc analysis was done using the Bonferroni test. Significant interactions were followed by repeated measures MANOVA applied to data corresponding to each emotion. To evaluate possible effects of the repetition (crossover design), a preliminary analysis was carried out to compare the performance in the behavioural task during the first experimental session with the performance during the second experimental session independently of treatment (order factor).

Subjective measures were also analysed with repeated measures MANOVA, with the factors of time (four levels) and treatment (two levels). Post-hoc comparisons were done with a paired $t$-test. Values of $p<0.05$ were considered significant.

\section{Results}

\section{Subjective measures}

The values of the four factors of the VAMS and of the global BSS index are shown in Table 1. A statistically significant main effect of treatment on subjective anxiety was identified $(F(1,11)=6.80 ; p=0.024)$. Post-hoc analyses showed that under the effects of diazepam, volunteers were less anxious at the end of the experimental session compared with the placebo session $(t=2.40, \mathrm{df}=11, p=0.035)$. Independently of treatment, volunteers reported increasing sedation (time factor $F(3,9)=4.78, p=0.029)$ throughout the experimental session. No significant difference between treatments was found in the remaining VAMS factors (cognitive impairment $F(1,11)=0.57 ; p=0.466$; discomfort $F(11)=1,05 ; p=0.327)$ or in the BSS global index $(F(1,8)=0.12, p=0.742)$.

\section{fMRI task}

Main effects of the tasks for fearful and angry faces, during the placebo sessions, drug effect (diazepam and placebo contrast) for each emotion and differences between emotions (fearful faces and angry faces contrasts) under both treatment conditions (diazepam and placebo), all of them as a result of whole-brain analysis, are presented as supplemental material (supplemental material, Tables S1 to S5).

These preliminary analyses have shown that diazepam attenuated the activations to fearful faces of several brain areas involved in emotional processing, including the right amygdala. Most of the activations to angry faces were attenuated by diazepam, but the activation of some areas (bilateral medial frontal gyrus, BA10; bilateral anterior cingulate gyrus, BA24 and 32; right fusiform gyrus, BA19; and right middle occipital gyrus, BA18) were enhanced by diazepam.

In agreement with the a priori hypothesis, region of interest analyses have shown that, independently of treatment, fearful faces activated the right amygdala, but no haemodynamic response was observed in the amygdala to angry faces.
The insula, the orbitofrontal cortex (OFC) and the anterior cingulate cortex (ACC) were activated by both fearful and angry faces.

Diazepam attenuated the activation of the right amygdala to fearful faces (Table 2 and Figure 1A), but no effect was observed on angry faces. Table 2 also shows that diazepam decreased the BOLD signal in the OFC (in right hemisphere) to fearful faces but not to angry faces.

Diazepam elicited a differential modulation on the anterior and posterior portions of the insula. In response to fearful faces, it attenuated the activation of bilateral anterior insula (Figure 1B), whereas in response to angry faces, it enhanced the activation of the left posterior insula (Figure 1C). In addition, diazepam increased the activation of the right ACC (BA 32) in response to fearful faces, but attenuated its activation, bilaterally, to angry faces.

No significant correlation between the accuracy in identifying the emotions of fear and anger in the behavioural task (described below) and the response amplitude to fearful and angry faces, respectively, during the fMRI task has been found. Higher levels of anxiety just before the scanner were associated with higher activation of right OFC to fearful faces, and of left ACC to angry faces. On the other hand, higher personality traits of anxiety were associated with less

Table 1. Mean and SEM of subjective measures (VAMS factors) and global index of the Bodily Symptoms Scale (BSS) under placebo and diazepam of 12 healthy male volunteers submitted to emotional tasks

\begin{tabular}{|c|c|c|c|c|c|c|}
\hline & \multicolumn{2}{|c|}{ Placebo } & \multicolumn{2}{|c|}{ Diazepam } & \multirow[b]{2}{*}{$t$} & \multirow[b]{2}{*}{$p$} \\
\hline & Mean & SEM & Mean & SEM & & \\
\hline \multicolumn{7}{|l|}{ Anxiety } \\
\hline Initial & 47.32 & 2.32 & 45.33 & 2.96 & 0.65 & 0.530 \\
\hline Pre-scanner & 49.13 & 2.09 & 45.67 & 2.78 & 1.47 & 0.168 \\
\hline Pos-scanner & 48.92 & 1.30 & 44.07 & 3.28 & 1.62 & 0.133 \\
\hline Final & 48.98 & 1.19 & 43.00 & 2.86 & 2.40 & $0.035^{*}$ \\
\hline \multicolumn{7}{|l|}{ Sedation } \\
\hline Initial & 49.85 & 2.21 & 44.71 & 1.81 & 1.73 & 0.112 \\
\hline Pre-scanner & 46.89 & 4.58 & 53.52 & 5.49 & 0.88 & 0.396 \\
\hline Post-scanner & 54.19 & 1.83 & 56.02 & 3.52 & 0.44 & 0.671 \\
\hline Final & 52.94 & 2.05 & 50.94 & 3.12 & 0.44 & 0.667 \\
\hline \multicolumn{7}{|c|}{ Cognitive impairment } \\
\hline Initial & 48.52 & 1.51 & 47.72 & 2.04 & 0.35 & 0.735 \\
\hline Pre-scanner & 51.26 & 2.37 & 55.64 & 1.92 & 1.47 & 0.169 \\
\hline Post-scanner & 50.11 & 0.62 & 52.59 & 2.98 & 0.83 & 0.426 \\
\hline Final & 51.14 & 1.17 & 50.85 & 3.11 & 0.08 & 0.937 \\
\hline \multicolumn{7}{|l|}{ Discomfort } \\
\hline Initial & 45.12 & 1.74 & 41.30 & 3.09 & 1.26 & 0.233 \\
\hline Pre-scanner & 46.29 & 2.38 & 45.33 & 1.81 & 0.31 & 0.760 \\
\hline Post-scanner & 45.28 & 1.37 & 42.74 & 3.48 & 0.69 & 0.502 \\
\hline Final & 47.83 & 0.67 & 43.36 & 3.38 & 1.46 & 0.171 \\
\hline \multicolumn{7}{|l|}{ BSS } \\
\hline Initial & 4.18 & 0.72 & 4.00 & 0.98 & 0.23 & 0.826 \\
\hline Pre-scanner & 3.64 & 0.94 & 5.45 & 1.05 & 1.29 & 0.231 \\
\hline Post-scanner & 5.58 & 1.14 & 6.42 & 1.24 & 0.73 & 0.480 \\
\hline Final & 5.50 & 1.55 & 6.27 & 1.18 & 0.24 & 0.818 \\
\hline
\end{tabular}

*Significant drug effect at $p<0.05$. 
activation in left ACC and left insula to angry faces. These data are shown in Table 3.

\section{Behavioural task}

Participants generally performed better in the second experimental session compared with the first (order factor, $F(1,10)=5.55 ; p=0.040)$. However, no significant interactions of the factor order with the factors emotion
$(F(5,6)=1.47 ; p=0.322)$, sex of the face $(F(1,10)=1.06$; $p=0.327)$ or treatment $(F(1,10)=0.40 ; p=0.542)$ were found.

The accuracy varied across the emotions $(F(5,7)=19.07$, $p=0,001$ ). Post-hoc analysis (Bonferroni) showed that, compared with aversive emotions, happiness and surprise were identified with higher accuracy. There was also a difference in the accuracy related to the sex of the faces: emotions were identified more precisely in female than in male faces $(F(1,11)=105.68, p<0.001)$.

Table 2. Maximally activated voxels in areas in which significant evoked activity was related to the covert recognition of fearful and angry faces blocks compared with the recognition of neutral faces blocks. Statistical threshold was set at $p<0.05$ corrected for multiple comparisons (FDR Corrected)

\begin{tabular}{|c|c|c|c|c|c|c|c|c|c|c|c|c|}
\hline \multirow[b]{2}{*}{ Brain Area } & \multirow[b]{2}{*}{ BA } & \multirow[b]{2}{*}{ Hemisphere } & \multicolumn{5}{|l|}{ Fearful faces } & \multicolumn{5}{|l|}{ Angry faces } \\
\hline & & & Drug effect & $p$-value & $x$ & Y & Z & Drug effect & $p$-value & $x$ & Y & Z \\
\hline \multirow[t]{2}{*}{ Amygdala } & & $\mathrm{R}$ & $\downarrow$ & 0.021 & 18 & -7 & -12 & - & & & & \\
\hline & & $\mathrm{L}$ & - & & & & & - & & & & \\
\hline \multirow[t]{2}{*}{ Orbitofrontal cortex } & 47 & $\mathrm{R}$ & $\downarrow$ & 0.001 & 38 & 21 & -6 & - & & & & \\
\hline & & L & - & & & & & - & & & & \\
\hline \multirow[t]{2}{*}{ Anterior Cingulate Gyrus } & 32 & $\mathrm{R}$ & $\uparrow$ & 0.029 & 11 & 25 & 23 & $\downarrow$ & 0.046 & 6 & 32 & 10 \\
\hline & & L & - & & & & & $\downarrow$ & 0.013 & -4 & 43 & -1 \\
\hline \multirow[t]{2}{*}{ Insula } & 13 & $\mathrm{R}$ & $\downarrow$ & 0.001 & 40 & 4 & 3 & - & & & & \\
\hline & & L & $\downarrow$ & 0.001 & -39 & -14 & 14 & $\uparrow$ & 0.025 & -41 & -19 & 8 \\
\hline
\end{tabular}

$\mathrm{BA}=$ Brodmann Area; $\mathrm{R}=$ Right; $\mathrm{L}=\mathrm{Left} ; \downarrow=$ attenuated the activation; $\uparrow=$ enhanced the activation.

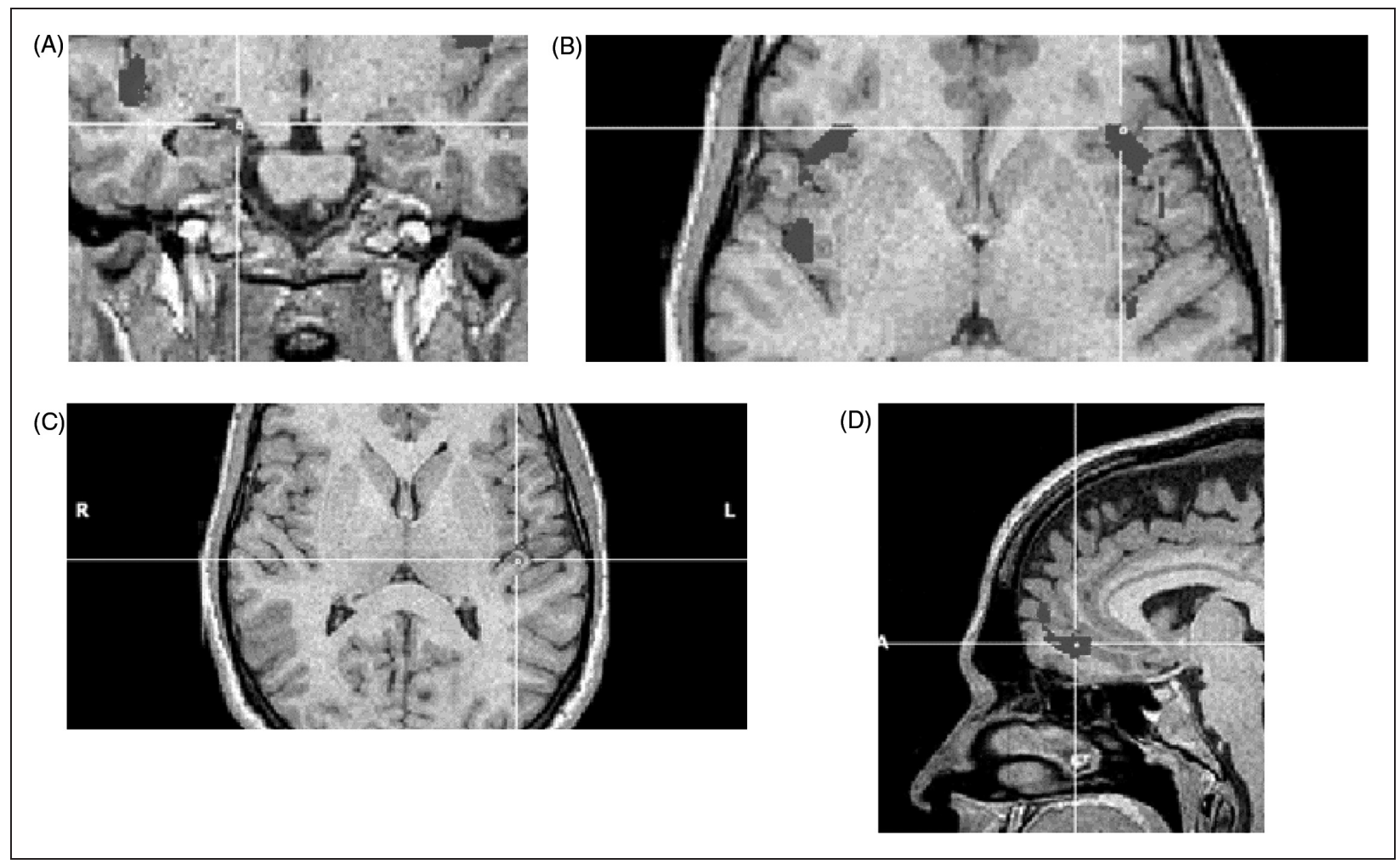

Figure 1. Brain areas modulated by diazepam (ingestion of $10 \mathrm{mg}$ ) during aversive face stimuli. A decrease is seen in the activation of the right amygdala (A) and the anterior bilateral insula (B) in response to fearful faces, and an increased modulation of the posterior left insula (C) and a decrease of the activation of left anterior cingulate gyrus (D) are observed in response to angry faces. 
Table 3. Correlation (correlation coefficient $r^{2}$ and its corresponding $p$-value) between activations in areas where a significant drug effect had been found and personality traits of anxiety (STAI-T); state of anxiety just before the scanner (VAMS); and the accuracy in the behavioural task to recognize fearful and angry faces

\begin{tabular}{|c|c|c|c|c|c|c|c|c|c|c|c|c|c|c|}
\hline & \multirow[b]{3}{*}{ BA } & \multirow[b]{3}{*}{$R / L$} & \multicolumn{6}{|c|}{ Fearful faces } & \multicolumn{6}{|c|}{ Angry faces } \\
\hline & & & \multicolumn{2}{|c|}{ Anxiety trait } & \multicolumn{2}{|c|}{ Anxiety state } & \multicolumn{2}{|c|}{ Accuracy } & \multicolumn{2}{|c|}{ Anxiety trait } & \multicolumn{2}{|c|}{ Anxiety state } & \multicolumn{2}{|c|}{ Accuracy } \\
\hline & & & $r$ & $p$ & $r$ & $p$ & $r$ & $p$ & $r$ & $p$ & $r$ & $p$ & $r$ & $p$ \\
\hline \multirow[t]{2}{*}{ Amygdala } & & $\mathrm{R}$ & 0.456 & 0.137 & -0.253 & 0.428 & -0.420 & 0.174 & - & - & - & - & - & - \\
\hline & & L & - & - & - & - & - & - & - & - & - & - & - & - \\
\hline \multirow[t]{2}{*}{ Orbitofrontal cortex } & 47 & $R$ & -0.129 & 0.689 & 0.651 & 0.022 & -0.022 & 0.946 & - & - & - & - & - & - \\
\hline & & L & - & - & - & - & - & - & - & - & - & - & - & - \\
\hline \multirow{2}{*}{$\begin{array}{l}\text { Anterior cingulate } \\
\text { gyrus }\end{array}$} & 32 & $\mathrm{R}$ & 0.328 & 0.298 & 0.245 & 0.443 & 0.410 & 0.185 & -0.410 & 0.198 & 0.313 & 0.323 & -0.126 & 0.696 \\
\hline & & L & - & - & - & - & - & - & -0.669 & 0.017 & 0.600 & 0.040 & -0.105 & 0.745 \\
\hline \multirow[t]{2}{*}{ Insula } & 13 & $R$ & -0.307 & 0.333 & 0.391 & 0.209 & 0.049 & 0.880 & - & - & - & - & - & - \\
\hline & & L & -0.162 & 0.615 & 0.384 & 0.218 & 0.223 & 0.487 & -0.629 & 0.029 & 0.559 & 0.059 & 0.261 & 0.412 \\
\hline
\end{tabular}

Bold figures indicate statistically significant correlations.

In the global analysis, no effect of diazepam on the recognition of facial expressions was found (treatment factor $F(1,11)=0.76 ; p=0.401$; treatment and emotion interaction $F(5,7)=1.26 ; p=0.376)$. However, an interaction between treatment, emotion and sex of the face nearly reached significance $(F(5,7)=3.95, p=0.051)$. The repeated measures MANOVA applied separately to data corresponding to each emotion showed a significant interaction between sex of the face and treatment for the emotion of fear $(F(1,11)=4.90, p=0.049)$. Figure 2 shows that diazepam impaired the recognition of fear in female faces. No significant interaction was found for the remaining emotions between treatment and sex.

\section{Discussion}

Regardless of pharmacological intervention, fearful faces activated the amygdala, which is a result that is consistent with other reports (Breiter et al., 1996; Fitzgerald et al., 2006; Whalen et al., 2001). More importantly, our results fulfil the prediction of the a priori hypothesis that fearful faces would be processed as a potential threat and, therefore, would activate the amygdala, a structure involved in the processing of ambiguous, but relevant stimuli (Harmer et al., 2006; Whalen, 1998).

Angry faces did not activate the amygdala, in contrast with reported evidence on the activation of the amygdala by facial expressions of anger (Fitzgerald et al., 2006; Hariri et al., 2000; Nomura et al., 2004). A possible explanation for the absence of amygdala activation in the present results may be that angry faces, in the paradigm adopted in this study, were interpreted as non-ambiguous stimuli representing a proximal threat, and were mainly processed by phylogenetically older structures of the brain (Deakin and Graeff, 1991) that were not assessed in this study, such as the periaqueductal gray matter. Moreover, there is also evidence for a positive correlation between personality traits of anxiety and activation of the amygdala, particularly on the right side, to attended angry faces, compared with both fearful and neutral faces (Ewbank et al., 2009). We could also allow that the absence of activation of the amygdala in response to angry faces observed in this study may be due to the relatively low anxiety levels of the participants assessed in this study.

A single dose of diazepam attenuated the activation of the right amygdala to fearful faces, which is a finding that is in agreement with results from animal studies that point to the amygdala as an important site of the anxiolytic action of drugs (Graeff et al., 1993; Menard and Treit, 1999). The result is also consistent with the high concentration of benzodiazepine receptors reported in the amygdala (Niehoff and Kuhar, 1983; Thomas et al., 1985). Nevertheless, no effect of diazepam was observed on the activation of the left amygdala. The right amygdala has been associated with an automatic and unconscious processing of fear, whereas the left amygdala may be involved in a broader and more complex analysis that helps to establish connections with cortical regions of the brain (Morris et al., 1998). In support of this view, it has been reported that electrical stimulation of the right amygdala during surgery of epileptic patients evokes only negative feelings, such as fear, anxiety and sadness, whereas simulation of the left amygdala causes both negative and positive (happiness) feelings (Lanteaume et al., 2007). This is also in agreement with the approach-withdrawn model proposed by Davidson (Davidson, 2003) about hemisphere asymmetry in emotional processing. According to this model, left-sided regions of the prefrontal cortex would be involved in approach-related behaviours, whereas right-sided regions of the prefrontal cortex would be engaged in behavioural inhibition. Right amygdala and right-sided prefrontal regions would be particularly involved with the occurrence of negative affects.

In contrast with the present results, it has been reported that lorazepam attenuates amygdala activation in both hemispheres (Paulus et al., 2005). This inconsistency may be due to methodological differences, since the effect of lorazepam was observed when negative emotions (anger and fear) were analysed together with a positive emotion (happiness), as well as in contrast with a sensorimotor task. In the present 


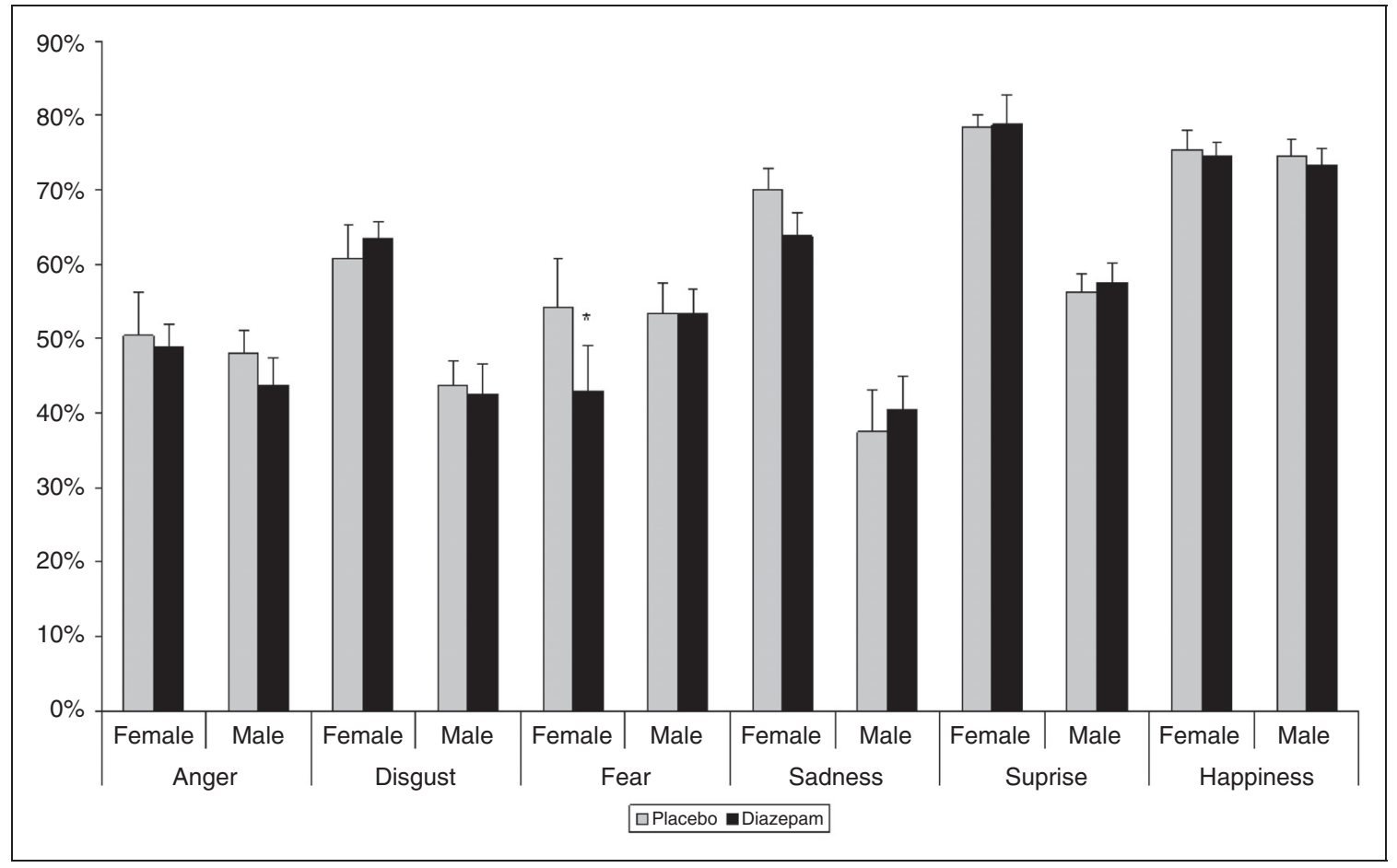

Figure 2. Effect of $10 \mathrm{mg}$ of diazepam (dark bars) compared with placebo (grey bars) on the accuracy of the recognition of six basic emotions in female and male facial expressions among 12 healthy male volunteers. The asterisks signal statistical significance of individual comparisons for each emotion $(p<0.05)$.

study, fear and anger were analysed independently, in contrast with a neutral facial expression. In fact, the modulation by diazepam of the neuronal response to faces of fear and anger resembles the results obtained with the intravenous administration of a low dose of the SSRI citalopram in a paradigm similar to that of the present study; citalopram attenuated the activation of the right amygdala to fearful faces with no effect on the response to angry faces (Anderson et al., 2007).

Considering the better response of GAD to benzodiazepines in comparison to that of PD (Nutt, 2005), our results are in agreement with the a priori hypothesis connecting fearful faces to potential threat (related to GAD) and angry faces to proximal threat (related to PD). It has been proposed that potential threat is mainly processed in the amygdala, whereas proximal threat is processed by phylogenetically older structures, such as the periaqueductal gray matter (Deakin and Graeff, 1991).

In addition to the attenuation of right amygdala activation, diazepam also bilaterally attenuated the activation of the anterior insular cortex to fearful faces, which is in agreement with a previous study showing that lorazepam attenuated activation of the insula by a face emotional task (Paulus et al., 2005). The insula has bidirectional connections with the amygdala (Augustine, 1996), receiving information about the salience of environmental stimuli and integrating this information with the possible consequences that these stimuli will have on the individual. The anterior insular cortex may provide information about future aversive bodily states associated with conditioned stimuli, and it may signal this information to brain areas that are critical to the allocation of attention and the execution of adaptive actions (Paulus and Stein, 2006). Our data suggest that the anxiolytic effect of benzodiazepines may also be related to an attenuation of emotional processes in the insula.

Interestingly, we found an enhancing effect of diazepam on the activation of the posterior insular cortex in response to angry faces. The posterior portions of the insula have been implicated in interoceptive processing, whereas the anterior portions are thought to be involved in the modulation and integration of cognitive and emotional processes (Craig, 2002, 2009). In addition, a recent fMRI study (Sabatini et al., 2009) has shown a dissociation between the anterior and posterior insular cortex in the processing of angry faces. The anterior insula was activated by angry faces either when they were paired with a painful stimulus (electrical stimulation of the wrist) or presented at a subliminal duration $(15 \mathrm{~ms})$ without an associated aversive stimulus. In contrast, the posterior insular cortex was activated only when the angry faces were paired with the electrical stimulation, and this result was in agreement with previously reported data pointing to the involvement of the posterior insular cortex in the processing of sensory information.

An enhancement of the activation of the posterior insula in response to disgusted faces has been previously reported, under the influence of a single dose of intravenous citalopram. This result was interpreted as an increase of the interoceptive processing by serotonin (Anderson et al., 2007). On the other hand, chronically administered escitalopram (3 weeks) attenuated the activation of the posterior insula, but not of the 
anterior insula, during a task of affective anticipation (Simmons et al., 2009). It may be hypothesized that the effect of diazepam observed in the present study on the posterior insular cortex could also be related to an increase of interoceptive processing which, in turn, could be involved in the paradoxical reactions to benzodiazepines described in clinical practice and commonly characterized by emotional release, excitement, and even hostility and rage (Mancuso et al., 2004).

The attenuation by diazepam of right orbitofrontal cortex activation to fearful faces is in agreement with the results obtained with lorazepam during negative emotional processing (Northoff et al., 2002). The present study has also found opposite effects of the drug on the activation of the ACC (BA 32) in response to the aversive faces. Whereas diazepam increased the activation of the right ACC to fear, it decreased the activation of the same brain area to anger. The ACC has been proposed to be part of a central executive system, integrating sensory and social information, evaluating the context and planning adaptive actions (Botvinick, 2007). The ACC is reciprocally interconnected with the anterior insula and with subcortical structures, such as the hypothalamus, periaqueductal gray matter, and amygdala (Ongur and Price, 2000). It is possible that the increase of the observed ACC activation by diazepam is related to the attenuation of the insula and the right amygdala to fearful faces through a top-down regulation of these structures (Quirk et al., 2003).

The impairment of the ability to identify fearful faces in the behavioural task is in agreement with reported data (Coupland et al., 2003; Zangara et al., 2002). However, these studies also found impairment in the identification of angry faces (Zangara et al., 2002) or of all the basic emotions studied (Coupland et al., 2003). These discrepancies may be related to dosage, since we have used a lower dose $(10 \mathrm{mg})$ of diazepam than that of the previous studies $(15 \mathrm{mg})$. Differences between the sexes may have also contributed to these data, as the previous studies have included both sexes in their samples, and we have used only male volunteers. Although this matter has not been completely clarified, there is growing evidence on sexual dimorphism in emotional processing (Guapo et al., 2009).

The present neuroimaging data should be interpreted cautiously, taking into account the small size of the sample and the analyses of but a few brain structures. Our data must thus be considered as an exploratory study. Also, we have not performed a functional connectivity analysis to explore the interaction among different brain regions. Since we have here used a passive placebo, we cannot be sure that the volunteers were not aware of the treatment that they were given, due to the likely recognition of the effects of the drug on their subjective state. Moreover, we were not able to correlate subjective, behavioural and neuroimaging data to the blood levels of diazepam, since the latter has not been assessed. In spite of these limitations, the present results add to previously reported evidence indicating that fMRI in combination with pharmacological challenges is a promising technique that offers a deeper understanding of the action in the CNS of widely used drugs that have well-known clinical efficacy.

Our data suggest that benzodiazepines can differentially modulate brain activation to aversive stimuli, depending on the stimulus features. In agreement with our working hypothesis, diazepam has attenuated amygdala activation to a stimulus viewed as a potential threat (fearful faces), whereas there was no effect on the haemodynamic response of the amygdala to angry faces, viewed as a proximal threat. This study has also shown that a single $10 \mathrm{mg}$ dose of diazepam selectively impaired the identification of fear expressed by female faces, and reduced subjective anxiety. The behavioural and subjective effects observed were preceded by an attenuation of the activation of the both right amygdala and bilateral anterior insula to fearful faces, evaluated by means of a covert task, which suggests that these structures play a role in the anxiolytic action of benzodiazepines.

\section{Funding}

This study was supported by Fundação de Amparo à Pesquisa do Estado de São Paulo (FAPESP-2007/03685-3). Cristina Marta Del-Ben and Draulio Barros de Araujo are supported by research fellowships from the Conselho Nacional de desenvolvimento Científico e Tecnológico (CNPq). Frederico Guilherme Graeff is supported by a research fellowship from the Fundação de Amparo ao Ensino, Pesquisa e Assistência do Hospital das Clínicas de Ribeirão Preto (FAEPA) and CNPq.

\section{Conflict of interest}

The authors declare that they have no conflict of interest.

\section{References}

Anderson IM, Del-Ben CM, McKie S, et al. (2007) Citalopram modulation of neuronal responses to aversive face emotions: a functional MRI study. Neuroreport 18: 1351-1355.

Augustine JR (1996) Circuitry and functional aspects of the insular lobe in primates including humans. Brain Res Brain Res Rev 22: 229-244.

Blair RJ and Curran HV (1999) Selective impairment in the recognition of anger induced by diazepam. Psychopharmacology (Berl) 147: 335-338.

Botvinick MM (2007) Conflict monitoring and decision making: reconciling two perspectives on anterior cingulate function. Cogn Affect Behav Neurosci 7: 356-366.

Breiter HC, Etcoff NL, Whalen PJ, et al. (1996) Response and habituation of the human amygdala during visual processing of facial expression. Neuron 17: 875-887.

Costafreda SG, Brammer MJ, David AS and Fu CH (2008) Predictors of amygdala activation during the processing of emotional stimuli: A meta-analysis of 385 PET and fMRI studies. Brain Res Rev 58: 57-70.

Coupland NJ, Singh AJ, Sustrik RA, Ting P and Blair R (2003) Effects of diazepam on facial emotion recognition. J Psychiatry Neurosci 28: 452-463.

Craig AD (2002) How do you feel? Interoception: The sense of the physiological condition of the body. Nat Rev Neurosci 3: 655-666.

Craig AD (2009) How do you feel - now? The anterior insula and human awareness. Nat Rev Neurosci 10: 59-70.

D'Hulst C, Atack JR and Kooy RF (2009) The complexity of the GABAA receptor shapes unique pharmacological profiles. Drug Discov Today 14: 866-875.

Davidson RJ (2003) Affective neuroscience and psychophysiology: Toward a synthesis. Psychophysiology 40: 655-665.

Davis M and Whalen PJ (2001) The amygdala: Vigilance and emotion. Mol Psychiatry 6: 13-34. 
Deakin JFW and Graeff FG (1991) 5-HT and mechanisms of defense. J Psychopharmacol 5: 305-315.

Del-Ben CM, Ferreira CA, Alves-Neto WC and Graeff FG (2008) Serotonergic modulation of face-emotion recognition. Braz J Med Biol Res 41: 263-269.

Del-Ben CM, Vilela JAA, Crippa JAS, Hallak JEC, Labate CM and Zuardi AW (2001) Confiabilidade Teste-Reteste da Entrevista Clínica Estruturada para o DSM-IV - Versão Clínica (SCIDCV) Traduzida para o Português. Rev Bras Psiquiatr 23: 156-159.

Ekman P and Friesen F (1976) Pictures of Facial Affect. Palo Alto, California: Consulting Psychology.

Ewbank MP, Lawrence AD, Passamonti L, Keane J, Peers PV and Calder AJ (2009) Anxiety predicts a differential neural response to attended and unattended facial signals of anger and fear. Neuroimage 44: 1144-1151.

First MB, Spitzer RL, Gibbon M and Williams JBW (1997) Structured Clinical Interview for DSM-IV-TR Axis I Disorders, Clinical Version ( $S C I D-C V)$. New York: Biometrics Research, New York State Psychiatric Institute.

Fitzgerald DA, Angstadt M, Jelsone LM, Nathan PJ and Phan KL (2006) Beyond threat: Amygdala reactivity across multiple expressions of facial affect. Neuroimage 30: 1441-1448.

Graeff FG and Del-Ben CM (2008) Neurobiology of panic disorder: From animal models to brain neuroimaging. Neurosci Biobehav Rev 32: 1326-1335.

Graeff FG, Silveira MC, Nogueira RL, Audi EA and Oliveira RM (1993) Role of the amygdala and periaqueductal gray in anxiety and panic. Behav Brain Res 58: 123-131.

Greenblatt DJ and Shader RI (1987) Pharmacokinetics of antianxiety agents. In: Meltzer HY (ed) Psychopharmacology: The Third Generation of Progress. New York: Raven Press, pp.1377-1386.

Guapo VG, Graeff FG, Zani AC, Labate CM, dos Reis RM and DelBen CM (2009) Effects of sex hormonal levels and phases of the menstrual cycle in the processing of emotional faces. Psychoneuroendocrinology 34: 1087-1094.

Hariri AR, Bookheimer SY and Mazziotta JC (2000) Modulating emotional responses: Effects of a neocortical network on the limbic system. Neuroreport 11: 43-48.

Harmer CJ, Mackay CE, Reid CB, Cowen PJ and Goodwin GM (2006) Antidepressant drug treatment modifies the neural processing of nonconscious threat cues. Biol Psychiatry 59: 816-820.

Kamboj SK and Curran HV (2006) Scopolamine induces impairments in the recognition of human facial expressions of anger and disgust. Psychopharmacology (Berl) 185: 529-535.

Lanteaume L, Khalfa S, Regis J, Marquis P, Chauvel P and Bartolomei F (2007) Emotion induction after direct intracerebral stimulations of human amygdala. Cereb Cortex 17: 1307-1313.

McNaughton N and Corr PJ (2004) A two-dimensional neuropsychology of defense: Fear/anxiety and defensive distance. Neurosci Biobehav Rev 28: 285-305.

Mancuso CE, Tanzi MG and Gabay M (2004) Paradoxical reactions to benzodiazepines: Literature review and treatment options. Pharmacotherapy 24: 1177-1185.

Menard J and Treit D (1999) Effects of centrally administered anxiolytic compounds in animal models of anxiety. Neurosci Biobehav Rev 23: 591-613.

Morris JS, Ohman A and Dolan RJ (1998) Conscious and unconscious emotional learning in the human amygdala. Nature 393: 467-470.

Murphy SE, Downham C, Cowen PJ and Harmer CJ (2008) Direct effects of diazepam on emotional processing in healthy volunteers. Psychopharmacology (Berl) 199: 503-513.

Niehoff DL and Kuhar MJ (1983) Benzodiazepine receptors: Localization in rat amygdala. J Neurosci 3: 2091-2097.
Nomura M, Ohira H, Haneda K, et al. (2004) Functional association of the amygdala and ventral prefrontal cortex during cognitive evaluation of facial expressions primed by masked angry faces: An event-related fMRI study. Neuroimage 21: 352-363.

Norris H (1971) The action of sedatives on brain stem oculomotor systems in man. Neuropharmacology 10: 181-191.

Northoff G, Witzel T, Richter A, et al. (2002) GABA-ergic modulation of prefrontal spatio-temporal activation pattern during emotional processing: A combined $\mathrm{fMRI} / \mathrm{MEG}$ study with placebo and lorazepam. J Cogn Neurosci 14: 348-370.

Nutt DJ (2005) Overview of diagnosis and drug treatments of anxiety disorders. CNS Spectr 10: 49-56.

Ongur D and Price JL (2000) The organization of networks within the orbital and medial prefrontal cortex of rats, monkeys and humans. Cereb Cortex 10: 206-219.

Parente AC, Garcia-Leal C, Del-Ben CM, Guimaraes FS and Graeff FG (2005) Subjective and neurovegetative changes in healthy volunteers and panic patients performing simulated public speaking. Eur Neuropsychopharmacol 15: 663-671.

Paulus MP and Stein MB (2006) An insular view of anxiety. Biol Psychiatry 60: 383-387.

Paulus MP, Feinstein JS, Castillo G, Simmons AN and Stein MB (2005) Dose-dependent decrease of activation in bilateral amygdala and insula by lorazepam during emotion processing. Arch Gen Psychiatry 62: 282-288.

Quirk GJ, Likhtik E, Pelletier JG and Pare D (2003) Stimulation of medial prefrontal cortex decreases the responsiveness of central amygdala output neurons. J Neurosci 23: 8800-8807.

Sabatini E, Penna SD, Franciotti R, et al. (2009) Brain structures activated by overt and covert emotional visual stimuli. Brain Res Bull 79: 258-264.

Simmons AN, Arce E, Lovero KL, Stein MB and Paulus MP (2009) Subchronic SSRI administration reduces insula response during affective anticipation in healthy volunteers. Int $J$ Neuropsychopharmacol 12: 1009-1020.

Spielberger CD (1983) Manual for the State/Trait Anxiety Inventory (Form Y): (Self Evaluation Questionnaire). Palo Alto, CA: Consulting Psychologists Press.

Talairach J and Tournoux P (1988) Coplanar Stereotactic Atlas of the Human Brain. Stuttgart: George Thieme Verlag.

Thomas SR, Lewis ME and Iversen SD (1985) Correlation of $[3 \mathrm{H}]$ diazepam binding density with anxiolytic locus in the amygdaloid complex of the rat. Brain Res 342: 85-90.

Whalen P (1998) Fear, vigilance, and ambiguity: Initial neuroimaging studies of the human amygdala. Curr Direct Psychol Sci 7: 12.

Whalen PJ, Shin LM, McInerney SC, Fischer H, Wright CI and Rauch SL (2001) A functional MRI study of human amygdala responses to facial expressions of fear versus anger. Emotion 1: $70-83$.

Young WS 3rd, Niehoff D, Kuhar MJ, Beer B and Lippa AS (1981) Multiple benzodiazepine receptor localization by light microscopic radiohistochemistry. J Pharmacol Exp Ther 216: 425-430.

Zangara A, Blair RJ and Curran HV (2002) A comparison of the effects of a beta-adrenergic blocker and a benzodiazepine upon the recognition of human facial expressions. Psychopharmacology (Berl) 163: 36-41.

Zilles K and Amunts K (2009) Receptor mapping: architecture of the human cerebral cortex. Curr Opin Neurol 22: 331-339.

Zuardi AW and Karniol I (1981) Estudo transcultural de uma escala de auto-avaliação para estados subjetivos. J Bras Psiquiatr 131: 4.

Zuardi AW, Guimaraes FS and Moreira AC (1993) Effect of cannabidiol on plasma prolactin, growth hormone and cortisol in human volunteers. Braz J Med Biol Res 26: 213-217. 